\title{
ON THE CLASSIFICATION OF NONCOMPACT SURFACES
}

\author{
BY \\ IAN RICHARDS( $(1)$
}

1. Introduction. In this paper we describe a complete topological classification of noncompact triangulable surfaces, and give a concrete model for an arbitrary surface, similar to the classical "normal form" for compact surfaces.

This classification of arbitrary surfaces depends on the well-known classification theorem for compact surfaces, and on the idea, frequently used in the theory of Riemann surfaces, of the "ideal boundary" of a surface. The ideal boundary is a totally disconnected, compact, separable space. For our purposes, we distinguish two nested closed subsets of this space, corresponding to portions of the surface which are of "infinite genus" and "infinitely nonorientable" respectively; thus our "ideal boundary" is really a nested triple of spaces.

Our first result is that, with certain fairly obvious qualifications, two surfaces are homeomorphic if and only if their ideal boundaries are topologically equivalent. This was originally discovered by Kerékjártó (see Kerékjártó [5, Chapter 5]). Kerékjártó's proof seems to contain certain gaps, so we have included an outline of a complete proof. (See in particular the remark following Proposition 3 in §3.)

In addition we prove the theorem, which so far as I know is new, that conversely, every nested triple of totally disconnected, compact, separable spaces occurs as the ideal boundary of some surface. At the same time, we show that every surface may be represented as a sphere, punctured by a finite or infinite number of discs and points, with the edges of the removed discs suitably identified. Thus we get a direct generalization of the classical representation theorem for compact surfaces.

2. Basic definitions. By a surface we mean a connected 2-dimensional manifold. Just as for any manifold, one can define the property of "orientability," which a given surface may or may not have.

A subset $A$ of a surface $S$ is said to be bounded in $S$ if its closure in $S$ is compact.

Received by the editors June 21, 1961 and, in revised form, January 21, 1962.

(1) The author wishes to thank Professor Lars V. Ahlfors for his encouragement and very valuable advice. This work was the main part of a doctoral dissertation submitted to the Faculty of Arts and Sciences at Harvard University in June 1960. This work was done while the author was a National Science Foundation Fellow. 
By a subsurface of a surface $S$, we mean a closed region in $S$ whose boundary in $S$ consists of a finite number of nonintersecting simple closed curves.

When dealing with a triangulable surface $S$ with some given triangulation $T$, we shall only allow subsurfaces which can be realized as subcomplexes of the complex formed by barycentrically subdividing $(S, T)$ a finite number of times. (We do not mention this point explicitly below, but in every case it is not difficult to verify that the subsurfaces which we describe can be chosen to be of this type. This allows us to use combinatorial methods freely.)

When referring to a subsurface by itself, we shall describe it as a bordered surface. (It is not difficult to give an intrinsic definition of "bordered surfaces," but we have no need for it.)

The Euler characteristic of any 2-complex is the number of its 0-simplexes, minus the number of its 1-simplexes, plus the number of its 2-simplexes. It is a topological invariant, that is, it is independent of the triangulation. (This is a consequence of the invariance of the homology groups; see e.g. Aleksandrov [2, Volume II, p. 71].)

We now give the fundamental classification theorem for compact bordered surfaces. (This theorem is due to Brahana [3].)

THEOREM. Two compact triangulable bordered surfaces are homeomorphic if and only if they both have the same number of boundary curves, the same Euler characteristic, and are either both orientable or else both nonorientable.

We define the reduced genus $g$ of a compact triangulable bordered surface $A$ with $q$ boundary curves and Euler characteristic $\chi$ by $g=1-\frac{1}{2}(\chi+q)$. It follows from the definition of $\chi$ that if $A$ and $A^{\prime}$ are compact bordered surfaces which are joined along $r$ common boundary curves, then (since the boundary curves have the same number of 0 and 1 simplexes) $\chi\left(A \cup A^{\prime}\right)=\chi(A)+\chi\left(A^{\prime}\right)$, and

$$
g\left(A \cup A^{\prime}\right)=g(A)+g\left(A^{\prime}\right)+(r-1) .
$$

REMARK. An orientable bordered surface of reduced genus $g$ is a sphere with $g$ "handles" and any number of "holes;" a nonorientable bordered surface of the same reduced genus has $2 g$ "cross caps," or equivalently, for any $k<g$, $k$ "handles" and $2 g-2 k$ "cross caps." (It is conventional to define the "genus" to be $g$ in the orientable case and $2 g$ in nonorientable case.)

A triangulable bordered surface is said to be planar if every compact subsurface in it is of reduced genus zero (or equivalently, of genus zero). (A surface without borders is planar or "schlichtartig" if and only if every Jordan curve separates it.)

3. The ideal boundary. We shall consider as our basic topic of investigation triangulable connected surfaces without borders. Such surfaces are clearly separable; it is known that any separable surface is also triangulable. (For a proof, see Ahlfors and Sario [1, Chapter 1, §46]; the theorem is due to Radó [6].) 
We begin by defining the ideal boundary.

Definition 1. A boundary component of a surface $S$ is a nested sequence $P_{1} \supset P_{2} \supset \cdots$ of connected unbounded regions in $S$ such that:

(a) the boundary of $P_{n}$ in $S$ is compact for all $n$;

(b) for any bounded subset $A$ of $S, P_{n} \cap A=\emptyset$ for $n$ sufficiently large.

We say that two boundary components $P_{1} \supset P_{2} \supset \cdots$ and $P_{1}^{\prime} \supset P_{2}^{\prime} \supset \cdots$ are equivalent if, for any $n$ there is a corresponding integer $N$ such that $P_{N} \subset P_{n}^{\prime}$ and vice versa. We let $p^{*}$ denote the equivalence class of boundary components containing $p=P_{1} \supset P_{2} \supset \cdots$.

Remark. Condition (a) above (also the corresponding restriction on $U$ in Definition 2 below) and the assumption that the sets $P_{n}$ are connected deserve emphasis; the ideal boundary gives a description of the way in which compact subsets of $S$ divide $S$ into unbounded components.

Definition 2. The ideal boundary $B(S)$ of a surface $S$ is the topological space having the equivalence classes of boundary components of $S$ as elements, and endowed with the following topology: for any set $U$ in $S$ whose boundary in $S$ is compact, we define $U^{*}$ to be the set of all boundary components $p^{*}$, represented by some $p=P_{1} \supset P_{2} \supset \cdots$, such that $P_{n} \subset U$ for $n$ sufficiently large; we take the set of all such $U^{*}$ as a basis for the topology of $B(S)$.

Definition 3. Let $p^{*}$, represented by $p=P_{1} \supset P_{2} \supset \cdots$, be a boundary component of $S$. We say that $p^{*}$ is planar and/or orientable if the sets $P_{n}$ are planar and/or orientable for all sufficiently large $n$.

REMARK. Of course it is necessary to verify that Definitions 2 and 3 do not depend on the representative $p$ chosen from the equivalence class $p^{*}$. The verification is trivial.

Following Definition 3, we shall consider the ideal boundary to be a nested triple of sets $B(S) \supset B^{\prime}(S) \supset B^{\prime \prime}(S)$, where $B(S)$ is the whole ideal boundary, $B^{\prime}(S)$ is the part which is not planar, and $B^{\prime \prime}(S)$ is the part which is not orientable.

It follows directly from the definitions that $B^{\prime}(S)$ and $B^{\prime \prime}(S)$ are closed subsets of $B(S)$.

EXAmple. If, for every compact subset $A$ of $S, S-A$ has at most $m$ unbounded components, and the number $m$ actually occurs for some $A$, then the ideal boundary of $S$ consists of $m$ discrete "points" (each of which may be planar, orientable but not planar, or nonorientable). The case $m=1$ deserves special mention, as many seemingly dissimilar noncompact surfaces fall into this category. If $m=0$ the surface is compact and vice versa.

DEFINITION 4. A bordered surface $S$ is of infinite genus and/or infinitely nonorientable if there is no bounded subset $A$ of $S$ such that $S-A$ is of genus zero and/or orientable.

Clearly an infinitely nonorientable surface is also of infinite genus.

On a nonorientable surface, a pair of "cross caps" is equivalent to a "handle" (as long as at least one cross cap is left). This fact leads to the following defini- 
tion, which is only significant for finitely nonorientable surfaces $S$ of infinite genus-in this case, $S-A$ is orientable for some bounded subset $A$ of $S$, and the number of cross caps in any bounded subsurface containing $A$ is always of the same parity.

Definition 5. We define four "orientability classes" of surfaces. Two classes are the orientable and the infinitely nonorientable surfaces; a surface which belongs to neither of these categories is said to be of odd or even nonorientability type according to whether every sufficiently large compact subsurface contains respectively an odd or an even number of "cross caps" (i.e., has half integral or integral reduced genus).

We now state, without proof, three important properties of the ideal boundary The first two are almost trivial. The third, which is basic for everything which follows, is proved in Ahlfors and Sario [1, Chapter 1, $\S 36$ and 37].

Proposition 1. Let $U$ and $V$ be subsets of a surface $S$ whose boundaries in $S$ are compact. Then (referring to the corresponding neighborhoods in $B(S)$ ), $[U \cup V]^{*}=U^{*} \cup V^{*}$, and $[U \cap V]^{*}=U^{*} \cap V^{*}$.

Proposition 2. If $S_{1}$ is a subsurface contained in a surface $S$, then the neighborhood $S_{1}^{*}$ in $B(S)$ contains nonplanar and/or nonorientable boundary components if and only if $S_{1}$ is of infinite genus and/or infinitely nonorientable. Similarly, $S_{1}^{*}$ is nonempty if and only if $S_{1}$ is unbounded.

Proposition 3. The ideal boundary of a separable surface is totally disconnected, separable, and compact.

REMARK. In the cases where the (homeomorphic) ideal boundaries of two surfaces are very complicated (e.g. equivalent to the Cantor set), the proof of Kerékjártó's theorem can involve serious combinatorial difficulties. These difficulties are very neatly handled using the compactness of the ideal boundary (see condition (5) in the proof of Theorem 1).

Finally we verify that what we have called "surfaces of infinite genus" deserve the name. This is intuitively obvious; a careful proof is easily given using the relationship between the genus and the Euler characteristic of a compact surface (see formula (1) in $\$ 2$ ).

Proposition 4. A bordered surface of infinite genus contains compact subsurfaces of arbitrarily large genus.

\section{Kerékjártó's Theorem.}

THEOREM 1. Let $S$ and $S^{\prime}$ be two separable surfaces of the same genus and orientability class. Then $S$ and $S^{\prime}$ are homeomorphic if and only if their ideal boundaries (considered as triples of spaces) are topologically equivalent.

Proof. Since, by definition, the ideal boundary is a topological invariant, 
the necessity is trivial. In proving the sufficiency, we shall assume that $S$ and $S^{\prime}$ are both infinitely nonorientable (and hence of infinite genus); it is easy to modify our proof to treat the general case.

The proof is based on a decomposition of $S$ and $S^{\prime}$ into compact subsurfaces (bordered by Jordan curves), and on the fact that a connected compact bordered surface is topologically determined by its orientability, genus, and the number of its boundary curves. Thus a compact surface of large genus contains a subsurface equivalent to any given (nonvacuously) bordered surface of lower genus and the same orientability.

Let $h$ be the given homeomorphism of the ideal boundary $B(S)$ onto $B\left(S^{\prime}\right)$. To construct the corresponding mapping $f$ from $S$ onto $S^{\prime}$, we shall represent $S$ and $S^{\prime}$ as unions of compact subsurfaces $A_{1} \subset A_{2} \subset \cdots$ and $A_{1}{ }^{\prime} \subset A_{2}{ }^{\prime} \subset \cdots$, each contained in the interior of the one following it, and, for each $n$, construct a homeomorphism $f_{n}$ of $A_{n}$ onto $A_{n}{ }^{\prime}$ so that $f_{n} \mid A_{n-1}=f_{n-1}$.

Furthermore, conditions (1) and (2) below (which mean that " $f_{n}$ commutes with $h$ ") will be imposed. We then define $f$ as the common extension of the $f_{n}$.

(1) For each $n$, there is exactly one simple closed curve $d(U)$ in the boundary of each component $U$ of $S-A_{n}$; similarly for the components $U^{\prime}$ of $S^{\prime}-A_{n}^{\prime}$.

(2) If $f_{n}(d(U))=d\left(U^{\prime}\right)$, then $h\left(U^{*}\right)=U^{\prime *}$. (Recall that, by Definition 2, $U^{*}$ is the neighborhood in $B(S)$ corresponding to $U$.)

We now take (totally unrelated) sequences $B_{1} \subset B_{2} \subset \cdots$ and $B_{1}^{\prime} \subset B_{2}^{\prime} \subset \cdots$ of bounded subsurfaces, each contained in the interior of its consequent, which cover $S$ and $S^{\prime}$ respectively and satisfy conditions (1) above (with $B_{n}$ replacing $\left.A_{n}\right)$ and (3) below.

(3) Every component of $S-B_{n}$ or $S^{\prime}-B_{n}^{\prime}$ is unbounded, either of genus zero or of infinite genus, and either orientable or infinitely nonorientable.

Condition (3) can be satisfied by arbitrarily large bounded $B_{n}$ because e.g. if a component $U$ of $S-B_{n}$ were of finite but not zero genus, we could (by Definition 4) remedy this by adding some bounded portion of $U$ to $B_{n}$. That condition (1) can always be satisfied as well seems geometrically evident; a careful proof could be constructed along the lines of Lemma (C) below.

The construction of the triples $\left(f_{n}, A_{n}, A_{n}^{\prime}\right)$ proceeds by induction (beginning with $\left.A_{0}=A_{0}^{\prime}=\varnothing\right)$. We require that $f_{n}, A_{n}$, and $A_{n}^{\prime}$ satisfy conditions (1), (2), and (3). Furthermore, we use a process which alternates in the following manner: when $n$ is even, we make $A_{n}$ large enough so that $B_{n} \subset A_{n}$, and then construct $A_{n}^{\prime}$ to fit; when $n$ is odd we interchange the roles of $S$ and $S^{\prime}$. Thus both $S$ and $S^{\prime}$ are covered by the unions of the sets $A_{n}$ and $A_{n}^{\prime}$.

Suppose we already have $\left(f_{n}, A_{n}, A_{n}^{\prime}\right)$. Assuming for the sake of definiteness that $n$ is even, we must construct $\left(f_{n+1}, A_{n+1}, A_{n+1}^{\prime}\right)$ so that $B_{n+1}^{\prime} \subset A_{n+1}^{\prime}$ and (1), (2), and (3) are still satisfied.

We first construct $A_{n+1}^{\prime}$. For some $M, A_{n}^{\prime}$ lies in the interior of $B_{M}^{\prime}$; we let $m=\max (n+1, M)$ and let $A_{n+1}^{\prime}=B_{m}^{\prime}$ (so that $B_{n+1}^{\prime} \subset A_{n+1}^{\prime}$ ). 
To construct $A_{n+1}$, we use the following:

(4) For $m$ sufficiently large, the closure of every component of $B_{m}-A_{n}$ is of reduced genus strictly greater than that of the corresponding component (see the remark below) of $A_{n+1}^{\prime}-A_{n}^{\prime}$, unless the latter component is of genus zero; each component of $B_{m}-A_{n}$ is nonorientable wherever the corresponding component of $A_{n+1}^{\prime}-A_{n}^{\prime}$ is.

REMARK. Recall that, by conditions (1) and (2) above, the homeomorphism $h$ gives a correspondence between the boundary curves of $S-A_{n}$ and those of $S^{\prime}-A_{n}^{\prime}$. Furthermore, by condition (1), the boundary of each component of $B_{m}-A_{n}\left(A_{n+1}^{\prime}-A_{n}^{\prime}\right)$ contains exactly one boundary curve of $S-A_{n}\left(S^{\prime}-A_{n}^{\prime}\right)$, as well as one or more boundary curves of $S-B_{m}\left(S^{\prime}-A^{\prime}{ }_{n+1}\right)$. If $A_{n}$ lies in the interior of $B_{m}$, every component of $S-A_{n}$ contains one nonvacuous component of $B_{m}-A_{n}$ (similarly for $A_{n}^{\prime}$ and $A_{n+1}^{\prime}$ ).

Proof of (4). Let $U$ and $U^{\prime}$ be corresponding components of $S-A_{n}$ and $S^{\prime}-A_{n}^{\prime}$, so that $h\left(U^{*}\right)=U^{\prime *}$. By hypothesis, $h\left(U^{*}\right)$ contains nonplanar and/or nonorientable boundary components if and only if $U^{*}$ does. Therefore, by Proposition 2 in $\$ 3$ and the condition (3) satisfied by $A_{n}$ and $A_{n}^{\prime}, U$ and $U^{\prime}$ are either both of infinite genus and/or infinitely nonorientable or both of genus zero and/or orientable. The result follows from Proposition 4 in $\$ 3$.

Now we consider condition (2). We need some notations: for any $m$, let $V_{1}^{m}, \cdots, V_{p}^{m}$ be the components of $S-B_{m}$; let $U_{1}^{\prime}, \cdots, U_{q}^{\prime}$ be the components of $S^{\prime}-A_{n+1}^{\prime}$; finally, let $\beta_{k}^{m}=\left[V_{k}^{m}\right]^{*}$ and $\alpha_{k}^{\prime}=\left[U_{k}^{\prime}\right]^{*}$ be the corresponding neighborhoods in $B(S)$ and $B\left(S^{\prime}\right)$. We get the weaker condition:

(5) For $m$ sufficiently large, every neighborhood $\beta_{i}^{m}$ in $B(S)$ is contained in the inverse image under $h$ of one of the neighborhoods $\alpha_{j}^{\prime}$ in $B\left(S^{\prime}\right)$.

Proof of (5). This follows from the compactness of $B(S)$. The sets $\beta_{k}^{m}, m=1,2, \cdots$, form a basis for the topology of $B(S)$, since any compact subset of $S$ is contained in $B_{m}$ for sufficiently large $m$ (see the remark following Definition 1). Since $A_{n+1}^{\prime}$ is bounded, $\left[A_{n+1}^{\prime}\right]^{*}=\varnothing$, and (by Proposition 1 in $\S 3$ ) the sets $\alpha_{j}^{\prime}$ cover $B\left(S^{\prime}\right)$. Thus every element of $B(S)$ is contained in some neighborhood $\beta_{i}^{r}$ which in turn lies in one of the sets $h^{-1}\left[\alpha_{j}^{\prime}\right]$; by the Heine-Borel property, a finite collection $Q$ of the $\beta_{i}^{r}$ covers $B(S)$. Letting $M$ be the maximum of the indices $r$ for $\beta_{i}^{r}$ in $Q$, it follows that (5) holds whenever $m \geqq M$ (since $r \leqq m$ implies $B_{r} \subset B_{m}$, and hence, for every $i$ and $j$, either $\beta_{i}^{m} \subset \beta_{j}^{r}$ or $\left.\beta_{i}^{m} \cap \beta_{j}^{r}=\varnothing\right)$.

Assume that $m$ is large enough so that both (4) and (5) hold. We shall then remove a certain part of each component of $B_{m}-A_{n}$ to get the desired set $A_{n+1}$, which must have the following properties:

(6) Each component of $A_{n+1}-A_{n}$ is homeomorphic to the corresponding component of $A_{n+1}^{\prime}-A_{n}^{\prime}$ (see the remark following (4) above).

(7) Each component of $S-A_{n+1}$ contains precisely those components of $S-B_{m}$ which correspond, according to (5), to some single component of $S^{\prime}-A_{n+1}^{\prime}$. 
In addition, $A_{n+1}$ must satisfy (1) and (3); we shall see that this is sufficient to allow us to find $f_{n+1}$.

We need the following properties of compact surfaces:

(A) If $\Sigma$ and $\Sigma^{\prime}$ are compact bordered surfaces which have the same reduced genus and orientability and the same number of boundary curves, then there are homeomorphisms $g$ of $\Sigma$ onto $\Sigma^{\prime}$ under which the boundary curves of $\Sigma$ correspond to those of $\Sigma^{\prime}$ in any preassigned manner.

(B) If $\Sigma$ is a compact bordered surface, $C$ is a simple closed curve in the border of $\Sigma$, and $\phi$ is any homeomorphism of $C$ onto itself, then $\phi$ can be extended to a homeomorphism of $\Sigma$ onto itself so that every curve in the border of $\Sigma$ is invariant under $\phi$.

(C) Let $\Sigma$ be a compact bordered surface of reduced genus $g$, and let $\Gamma_{1}, \cdots, \Gamma_{p}$ be a partition of the set of its boundary curves. Then, for any $k \leqq \max \left(g-\frac{1}{2}, 0\right)$, there exist $p-1$ nonintersecting simple closed curves in $\Sigma$ which divide $\Sigma$ into $p$ components $U_{1}, \cdots, U_{p}$ so that $\Gamma_{i} \subset U_{i}$ for all $i, U_{1}$ is of reduced genus $k$, and $U_{i}$ is of genus zero for $1<i<p$. If $\Sigma$ is nonorientable and $k$ is integral, then $U_{1}$ can be made either orientable or nonorientable.

RemARKS. (A) is simply the classical classification theorem for compact bordered surfaces (see $\$ 2$ above). (B) is trivial if the mapping $\phi$ preserves orientation on the curve $C$; if $\phi$ reverses orientation, we simply "turn the surface inside out" (reflect the normal polygon in a line), then proceed as before. (C) is tedious. But it is clear that a proof could be written down, using the normal form of $\Sigma$ as a polygon in the plane (punctured by discs which, by (A), can be arranged any way we please). The inequality $k \leqq g-\frac{1}{2}$ instead of $k \leqq g$ is needed only where we wish to make $U_{1}$ orientable when $\Sigma$ is not.

We now return to the proof of Theorem 1 . For convenience, in the remainder of this proof we shall write e.g. $B_{m}-A_{n}$, where we actually mean its closure in $S$, which consists of one or more connected compact subsurfaces of $S$.

By condition (4) above, each component of $B_{m}-A_{n}$ is of sufficiently large reduced genus and the right orientability so that, using Lemma (C), we can retract $B_{m}$ to a subsurface $A_{n+1}$ containing $A_{n}\left(B_{m} \supset A_{n+1} \supset A_{n}\right)$ so that the reduced genus and orientability of each component of $A_{n+1}-A_{n}$ exactly matches that of the corresponding component of $A_{n+1}^{\prime}-A_{n}^{\prime}$ (see the remark following (4)). (The components of $A_{n+1}-A_{n}$ correspond to $U_{1}$ and the components of $B_{m}-A_{n+1}$ to the other $U_{i}$ in Lemma (C).)

Recall that, by condition (5), several components of $S-B_{m}$ correspond (under $h$, as above) to one component of $S^{\prime}-A_{n+1}^{\prime}$. In retracting $B_{m}$ to $A_{n+1}$, exactly the right components of $S-B_{m}$ must be joined together inside of $B_{m}$ so that the components of $S-A_{n+1}$ satisfy conditions (1) and (7); again Lemma (C) shows that this is possible. Finally, within each component of $B_{m}-A_{n}$, we can have every component except one of $B_{m}-A_{n+1}$ (corresponding to $U_{i}, 1<i<p$, 
in Lemma (C)) of genus zero; and the extra component (if any) may be added to the part of $S-B_{m}$ which is already of infinite genus or infinitely nonorientable, so that $A_{n+1}$ satisfies (3).

To construct $f_{n+1}$, we use the classification theorem (A) to give a homeomorphism $g$ of $A_{n+1}-A_{n}$ onto $A_{n+1}^{\prime}-A_{n}^{\prime}$; since $f_{n}$ satisfies (2) and $A_{n+1}$ satisfies (7), we may assume that $g^{-1} f_{n}$ maps each curve in the boundary $d\left(A_{n}\right)$ onto itself, and that $g$ satisfies (2) on $d\left(A_{n+1}\right)$. We let $\phi=g^{-1} f_{n}$ on $d\left(A_{n}\right)$, and extend $\phi$ to $A_{n+1}-A_{n}$ as in (B) (here we use the fact that there is only one curve in the boundary of each component of $\left.S-A_{n}\right)$. Finally, we set $f_{n+1}(x)=g \phi(x)$ for $x \in A_{n+1}-A_{n}, f_{n+1}(x)=f_{n}(x)$ for $x \in A_{n}$; then $f_{n+1}(x)$ is continuous on $d\left(A_{n}\right)$ and satisfies (2) on $d\left(A_{n+1}\right)$.

Thus $\left(f_{n+1}, A_{n+1}, A_{n+1}^{\prime}\right)$ satisfies all the necessary conditions. This completes the proof of Theorem 1 .

5. Construction of a surface with a given ideal boundary. We recall that the ideal boundary of any surface is a nested triple of compact, separable, totally disconnected spaces. In this section we show that any such triple actually occurs as the ideal boundary of some surface. We do this by explicitly constructing the surface as a sphere, punctured by a certain set of points and open discs, with specified boundary identifications for the discs.

A totally disconnected space $X$ is of topological dimension 0 , and by a wellknown theorem (see Hurewicz and Wallman [4, Chapter 5, §3]), any compact separable space of topological dimension $n$ may be imbedded in $2 n+1$ dimensional Euclidean space. Thus $X$ may be imbedded in the real line. But it is easy to represent a compact totally disconnected subset of the real line as a subset of the Cantor set. Thus we have

Proposition 5. Any compact, separable, totally disconnected space $X$ is homeomorphic to a subset of the Cantor set.

REMARK. It is not too difficult to prove Proposition 5 directly. The idea is to form a basis for the topology of $X$ composed of sets which are both open and closed in $X$, and from this to extract a basis equivalent to the natural basis for a subset of the Cantor set.

THEOREM 2. Let $(X, Y, Z)$ be any triple of compact, separable, totally disconnected spaces with $Z \subset Y \subset X$. Then there is a surface $S$ whose ideal boundary $\left(B(S), B^{\prime}(S), B^{\prime \prime}(S)\right.$ ) (see the paragraph following Definition 3) is topologically equivalent to the triple $(X, Y, Z)$.

Proof. By Proposition 5 above, we may assume that $X$ is a subset of the Cantor set, which we imbed in the one point compactification of the plane (a sphere) as the set of all points $(x, 0)$ such that $0 \leqq x \leqq 1$ and $x$ admits a triadic expansion which does not involve the digit 1 . 
Let $D^{\prime}$ be the collection of all closed discs in the $x y$ plane whose diameters are the intervals in the $x$ axis $\left[(n-1 / 3) / 3^{m},(n+4 / 3) / 3^{m}\right], 0 \leqq n \leqq 3^{m}$, where $n$ is an integer which admits a triadic expansion free from 1's; let $D$ be the subcollection consisting of all discs in $D^{\prime}$ which contain at least one point of $X$. Then $D$ determines a basis for the topology of $X$. The lattice, under inclusion, of sets in the collection $D$ has the following properties, which we shall use below:

(1) Any two discs in $D$ are either disjoint or else one contains the other.

(2) The intersection of the discs in any infinite ordered chain of discs in $D$ consists of exactly one point of $X$ (because $X$ is compact; furthermore, the set of discs containing any point of $X$ forms an ordinary sequence under the inclusion ordering).

Let $P^{+}$and $P^{-}$be respectively the half planes $y>0$ and $y<0$. For each disc $K$ in $D$, let $K^{\prime}$ and $K^{\prime \prime}$ be the two largest discs in $D$ properly contained in $K$. (Then every disc in $D$ properly contained in $K$ is contained in $K^{\prime}$ or $K^{\prime \prime}$.)

For every disc $K$ in $D$, we choose two circles $C^{+}(K)$ and $C^{-}(K)$, each contained in the interior of $K$, such that:

(a) $C^{+}(K) \subset P^{+}$, and $C^{-}(K) \subset P^{-}$;

(b) $C^{+}(K)$ and $C^{-}(K)$ do not intersect either $K^{\prime}$ or $K^{\prime \prime}$;

(c) $C^{+}(K)$ and $C^{-}(K)$ are symmetric with respect to the $x$ axis.

Then no two distinct circles $C^{ \pm}(K)$ intersect.

We now construct $S$ as a "double" of the sphere with the points in $X$ and the interiors of some of the circles $C^{ \pm}(K)$ removed. First we fill in (or simply ignore) the circles $C^{ \pm}(K)$ for all $K \in D$ for which $K \cap Y=\varnothing$, and remove the interiors of $C^{ \pm}(K)$ for all $K \in D$ for which $K \cap Y \neq \emptyset$. If $K \cap Y \neq \emptyset$ but $K \cap Z=\varnothing$, then we identify the boundaries of $C^{+}(K)$ and $C^{-}(K)$ by reflecting $C^{+}(K)$ in the $x$ axis (preserving orientation in $S$ ). If $K \cap Z \neq \varnothing$, then we identify $C^{+}(K)$ and $C^{-}(K)$ by translating $C^{+}(K)$ onto $C^{-}(K)$ (reversing orientation in $S$ ).

We shall show that the ideal boundary of $S$ is equivalent to the triple $(X, Y, Z)$. It follows from (1) and (2) above that any point in $X$ can be uniquely represented as the intersection of the sets in a maximal ordered chain in the lattice of sets in $D$. By comparison with Definition 1 , we see that every such maximal chain represents an (abstract) boundary component of $S$. This defines a mapping from $X$ into $B(S)$.

Since $X-Y$ and $X-Z$ are open subsets of $X$, every point $p$ in $X-Y$ is contained in some disk $K \in D$ such that $K \cap Y=\varnothing$, and similarly for every $p \in X-Z$. Hence the subsets $Y$ and $Z$ of $X$ correspond precisely to the maximal chains which represent nonplanar and nonorientable boundary components of $S$ (see Definition 3).

It remains to show that every boundary component of $S$ is equivalent to one of those which corresponds to a point in $X$, and that the resulting correspondence between $B(S)$ and $X$ is a homeomorphism. The sets $K \in D$ form a basis for the 
topology of $X$, and it follows easily from Proposition 1 that the corresponding sets $K^{*}, K \in D$, form a basis for the topology of $B(S)$. Thus it is clear that the correspondence between maximal chains in $D$ and points in $X$ gives a homeomorphism of $X$ into $B(S)$.

We now show that this homeomorphism is onto. Since $B(S)$ is Hausdorff, every point $p^{*}$ in $B(S)$ is the intersection of some infinite collection [p] of sets $K^{*}$ from the basis $\left(K^{*} \mid K \in D\right)$ for the topology of $B(S)$. (If $p^{*}$ is isolated in $B(S)$, we can still take an infinite number of different sets $K$, although almost all of the $K^{*}$ will coincide.) By condition (1), the collection $p_{1}=\left(K \mid K^{*} \in[p]\right)$ of sets in the plane is nested and represents a boundary component $p_{1}^{*}$ of $B(S)$; but then $p_{1}^{*}$ must be equal to $p^{*}$. By (2), the intersection of all the sets in $p_{1}$ is a single point $p^{\prime}$ in $X$. Finally $p^{\prime}$ corresponds to $p_{1}^{*}$ under the homeomorphism of $X$ into $B(S)$, and $p_{1}^{*}=p^{*}$. This proves Theorem 2 .

6. A representation theorem for noncompact surfaces. The ideal boundary of any surface $S^{\prime}$ is a nested triple $X \supset Y \supset Z$ of compact, separable, totally disconnected spaces. By Theorem 1 , the sets $(X, Y, Z)$ almost determine $S^{\prime}$, and in proving Theorem 2 we found a surface $S$, constructed by taking a "double" of a modified sphere, having $(X, Y, Z)$ as its ideal boundary.

This almost implies that every surface $S^{\prime}$ has a structure like $S$. In fact, according to Theorem 1, we need only consider possible variations in the genus and orientability class of $S$ and $S^{\prime}$. But, by Proposition 2 in $\S 3$, if either of these surfaces has infinite genus or degree of nonorientability, then the assumption that these invariants be the same is redundant (since it is contained in the assumption that the ideal boundaries contain nonplanar or nonorientable boundary components). Since it is possible to vary the genus or degree of nonorientability in the finite case by adding or subtracting a finite number of "handles" or "cross caps," we get the following result.

THEOREM 3. Every surface is homeomorphic to a surface formed from a sphere $\Sigma$ by first removing a closed totally disconnected set $X$ from $\Sigma$, then removing the interiors of a finite or infinite sequence $D_{1}, D_{2}, \cdots$ of nonoverlapping closed discs in $\Sigma-X$, and finally suitably identifying the boundaries of these discs in pairs. (It may be necessary to identify the boundary of one disc with itself to produce an odd "cross cap.") The sequence $D_{1}, D_{2}, \cdots$ "approaches $X$ ' in the sense that, for any open set $U$ in $\Sigma$ containing $X$, all but a finite number of the $D_{i}$ are contained in $U$.

(We remark that, in the orientable case, when the ideal boundary contains no planar components, we could also represent the surface as the standard "double" of a punctured sphere.)

7. Planar surfaces. We show that the (abstract) boundary components of a region in the sphere correspond in a natural way to the ordinary components 
of the boundary of that region. (Recall that an abstract boundary component of such a region is a nested sequence of connected subsets whose boundaries inside the region are bounded away from the boundary of the region itself in the sphere.)

THEOREM 4. Let $\Sigma$ be a sphere and let $S$ be an open connected region in $\Sigma$. Let $P_{1} \supset P_{2} \supset \cdots$ represent an (abstract) boundary component of $S$; let $\bar{P}_{n}$ denote the closure of $P_{n}$ in $\Sigma$ (not in $S$ ), and let $A=\bar{P}_{1} \cap \bar{P}_{2} \cap \cdots$. Then the set $A$ is equal to one component of the boundary $B$ of $S$ in $\Sigma$. Every component $A^{\prime}$ of $B$ corresponds in this way to some abstract boundary component of $S$. Finally, a sequence $A_{1}, A_{2}, \cdots$ of components of $B$ converges (in the sense of Definition 2) to a component $A$ if and only if $\lim _{\inf _{n \rightarrow \infty}}\left(|x-y| \mid x \in A_{n}, y \in A\right)=0$.

Proof. Trivial compactness arguments show that $A$ is connected and nonempty, that any neighborhood of $B$ in $\Sigma$ contains $\bar{P}_{n}$ for $n$ sufficiently large, and that different components of $B$ are contained in disjoint neighborhoods in $\Sigma$ and thus correspond to different abstract boundary components of $S$. The condition for convergence of the components $A_{n}$ is also trivial.

What is harder to prove is that $A$ is not a proper subset of one of the components $A^{\prime}$ of $B$, and that every $A^{\prime}$ occurs as some set of the form $A$. This follows once we show that, for arbitrarily small connected neighborhoods $U$ of $A^{\prime}$ in $\Sigma, U \cap S$ is connected. To do this, we apply the following lemma to the sets $U$ and $S$.

LEMMA. If $E$ and $F$ are connected open subsets of the sphere $\Sigma$ whose boundaries in $\Sigma$ do not intersect, then $E \cap F$ is connected.

Proof of lemma. This depends on the fact that $\Sigma$ is simply connected. Suppose that the hypotheses of the lemma are satisfied but $E \cap F$ is the union of two nonempty disjoint open sets $G$ and $H$. Then we can construct a nonsingular two sheeted covering surface $\Sigma^{*}$ of $\Sigma$, contradicting the monodromy theorem. To do this, we put a pair of sheets $G_{1}, G_{2}$ and $(\Sigma-G)_{1},(\Sigma-G)_{2}$ above the sets $G$ and $\Sigma-G$ in $\Sigma$, and attach $G_{i}(i=1,2)$ to $\left\{(\Sigma-G)_{i} /(\Sigma-G)_{i \pm 1}\right\}$ along the (nonintersecting) boundaries of $G$ in $\{E / F\}$ respectively. Since $E$ and $F$ are connected and intersect in $H, \Sigma^{*}$ is connected. This proves the lemma.

\section{BIBLIOGRAPHY}

1. L. V. Ahlfors and L. Sario, Riemann surfaces, Princeton Univ. Press, Princeton, N. J., 1960.

2. P. Aleksandrov, Combinatorial topology. I, II, Graylock, Rochester, N. Y., 1956, 1957.

3. H. R. Brahana, Systems of circuits on two-dimensional manifolds, Ann. of Math. 23(1921), 144-168.

4. W. Hurewicz and H. Wallman, Dimension theory, Princeton Univ. Press, Princeton, N. J., 1941.

5. B. Kerékjártó, Vorlesungen über Topologie. I, Springer, Berlin, 1923.

6. T. Radó, Über den Begriff der Riemannschen Fläche, Acta Litt. Sci. Szeged 2 (1925), 101-121.

Massachusetts Institute of Technology, Cambridge, Massachusetts 